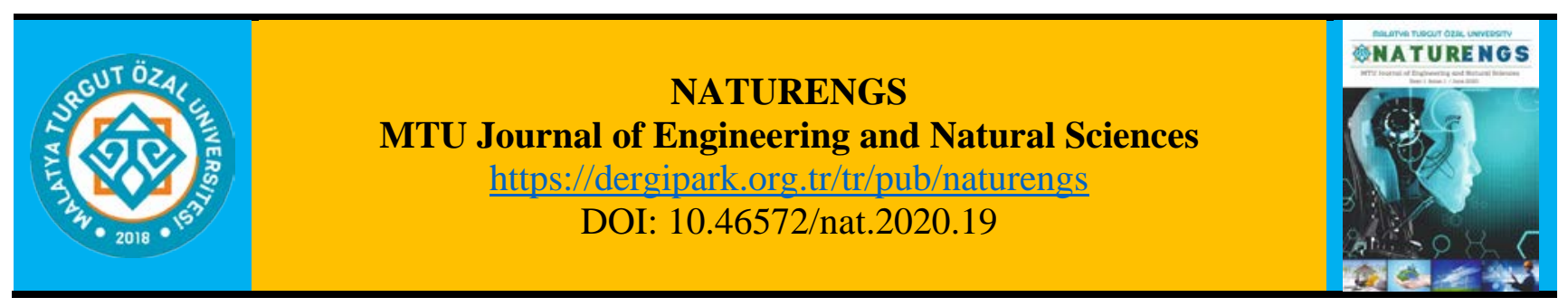

\title{
The Effect of Align Parameter on Scattering Parameter for Pseudomorphic High Electron Mobility Transistor
}

\author{
Gökhan SATILMIŞ* \\ Electric and Electronic Engineering, Engineering and Architecture, Muş Alparslan University, Muş, Turkey
}

(Received: 06.12.2020; Accepted: 30.12.2020)

\begin{abstract}
In this paper, various physical device simulation of pseudomorphic High Electron Mobility Transistors are realized to show the effect of aligning parameter on scattering parameters. S parameters are complex numbers, so both real and imaginer part of scattering parameters are plotted concerning frequency at different align parameters. In all figures, a brief explanation about the change of the $\mathrm{S}$ parameter for frequency and align parameter is provided. The effect of the align parameter, which has a value of 0.4 differs from other align parameter values such as $0.45,0.5,0.55$ and 0.6 on scattering parameters of the transistor structure.
\end{abstract}

Keywords: PHMET, Scattering parameters, Align parameter, Device simulation.

\section{INTRODUCTION}

The name HEMT transistor is referred to as High Electron Mobility Transistor. The transistor is a type of field-effect transistor. The HEMT transistor is structured with a narrow channel, which enables operating at high frequencies. The device structure incorporates a junction of two materials with different energy band gaps. The development of HEMT transistor goes back to the 1980s, when its cost is high, so the applications of the transistor are limited. Nowadays, the production cost of HEMT transistor is low comparing to the past, so the application of the transistor are common in communication technologies such as microwave radio communication, mobile communication, and RF (Radio Frequency) design circuits [1-4]. The pseudomorphic High Electron Mobility Transistor (pHEMT) [5-9] is modeled and used for various applications such as low noise amplifier, power amplifier, oscillator or mixers. Transistors are considered as two-port networks for designing circuits, and scattering (S) parameters are the main parameters for calculating the performance of the design circuits. In other words, transistors are modeled as two-port networks, where the input port gate electrode and the output port is a drain electrode. S parameters of these two-port networks affect the performance of the designed circuits. S parameters are modeled with various optimization algorithms and neural networks [10]. Physical parameter based modeling of S parameter is realized in this study $[11,12]$ using symbolic regression, and novel classification algorithms. The power gain and stability of the microwave circuit can be defined by S parameters [13-17]. $\mathrm{S}$ parameters are dependent on various parameters such as bias condition parameters, device parameters and frequency. The effect of the align parameter on the S parameter is studied in this paper. 
Bandgap, which is the difference between two material energy bands cause conduction and valence band discontinues. The distribution of bandgap difference on conduction and valence band has an impact on charge transport, and so forth $\mathrm{S}$ parameters of the device. Align parameter is defined as a portion of the energy bandgap difference, that is applied to the conduction band of the transistor. For instance, \%80 percent of the bandgap difference can be assigned to material 1 and material 2 to the conduction band offset by setting the align parameter to 0.8 .

In this study, the effect of the align parameter on S parameters is expressed from $1 \mathrm{GHz}$ to 100 $\mathrm{GHz}$ for pHEMT structure. The align parameter is ranged from 0.4 to 0.6 with a step of 0.5 . This study is realized using a physical device simulator. S parameters are complex numbers, so real and imaginer parts of S parameters are calculated and plotted on a cartesian coordinate system.

\section{DEVICE SIMULATION}

A physics-based device simulator is chosen in this study. Physics-based device simulators calculate the electrical characteristics of the structure given bias conditions. The simulator uses a numerical method such as Newton, Gummel or Block method. [18]. As a result, the device can be modeled in AC for S parameter analysis. The major advantages of a physical-based device simulator are being predictive, providing insight into the structure. Moreover, physicsbased device simulation is compared to actual experiments, it has many advantages such as being faster, being cheaper and providing information, that requires extensive work using experimental work.

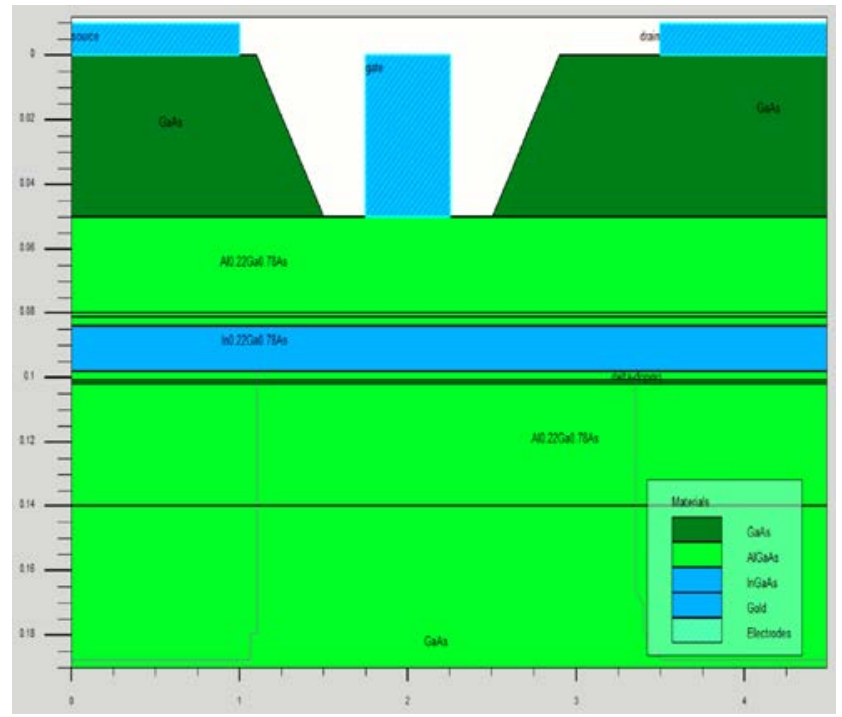

Figure 1. pHEMT Structure [19]

In Figure 1, the pHEMT structure is shown and the architecture of the transistor is referenced in [19]. Source, gate and drain electrodes are placed at the top of the structure. Source and drain electrodes are in contact with GaAs material, whereas the gate electrode in contact with $\mathrm{Al}_{0.22}$ Ga0.78As material. Delta doping, which is a very thin layer is used between In0.22Ga0.78As material. Al $0.22 \mathrm{Ga} 0.78 \mathrm{As}$ material is placed on GaAs substrate, and the device is symmetrical to In $0.22 \mathrm{Ga} 0.78 \mathrm{As}$ material region if the electrodes and their contact with GaAs substrate are omitted. 
The transistor is considered as two-port networks, where the gate electrode is the input port, and the drain electrode is the output port. Small signal analysis of the transistor is realized from $1 \mathrm{GHz}$ to $100 \mathrm{GHz}$ to exhibit the entire potential use on various applications.

\section{RESULTS AND DISCUSSION}

5 different physical device simulations are realized for showing the effect of the align parameter. The values of these parameters are listed in Table 1. Drain voltage and gate voltage are set to 2(V) and $0(\mathrm{~V})$, respectively. The effect of the align parameter on S parameters is plotted both real and imaginer values on cartesian coordinates, separately. The effect of the align parameter on $\mathrm{S}$ parameters is plotted from Figure 2 to Figure 9. In all figures, the absolute value of real and imaginer part of $S$ parameters are analyzed.

Table 1. Align Parameter Range

\begin{tabular}{|l|l|}
\hline Align Parameter & Frequency \\
\hline 0.4 & $1,2, \ldots 100 \mathrm{GHz}$ \\
\hline 0.45 & $1,2, \ldots 100 \mathrm{GHz}$ \\
\hline 0.5 & $1,2, \ldots 100 \mathrm{GHz}$ \\
\hline 0.55 & $1,2, \ldots 100 \mathrm{GHz}$ \\
\hline 0.6 & $1,2, \ldots 100 \mathrm{GHz}$ \\
\hline
\end{tabular}

In Figure 2, the real part of $S_{11}$ is plotted from $1 \mathrm{GHz}$ to $100 \mathrm{GHz}$ with all aligned parameters listed in Table 1. It can be seen in Figure 1 that the real part of $S_{11}$ steadily decreases as the frequency increase. The minimum value is around $20 \mathrm{GHz}$, which makes a good return loss value. All align parameter values has almost no effect on the real part of the $\mathrm{S}_{11}$ parameter.

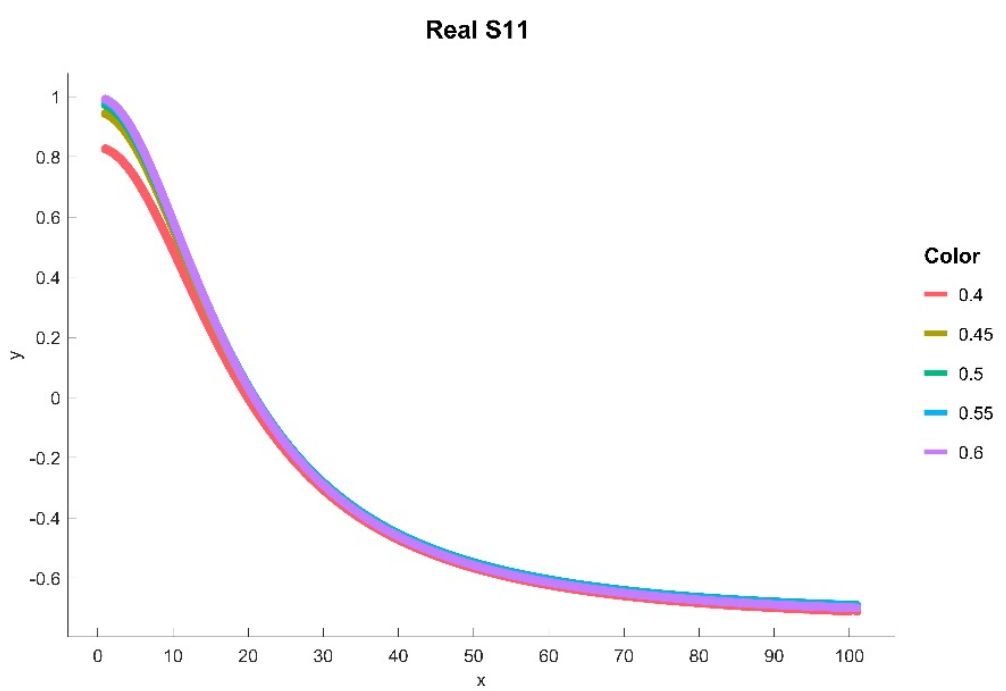

Figure 2. The effect of aligning parameter on the real part of the $S_{11}$ parameter

In Figure 3, the imaginer part of $S_{11}$ is plotted from $1 \mathrm{GHz}$ to $100 \mathrm{GHz}$ with all aligned parameters listed in Table 1. It can be seen in Figure 2 that the imaginer part of $\mathrm{S}_{11}$ increments until frequency reaches $20 \mathrm{GHz}$, and decrement again. All align parameter values has almost no effect on the imaginer part of the $\mathrm{S}_{11}$ parameter. 


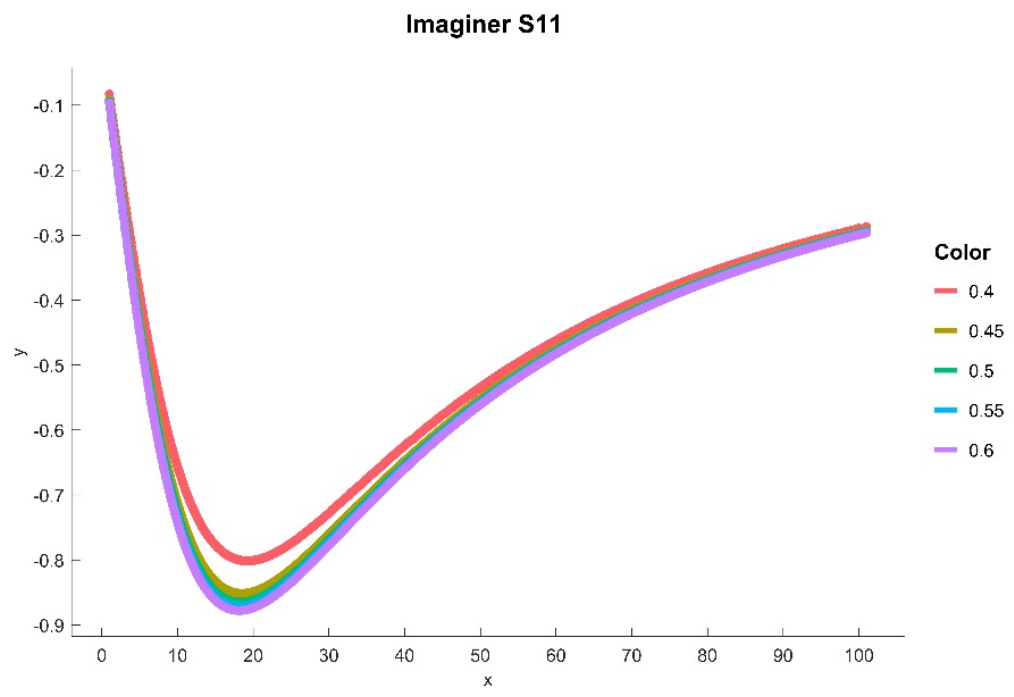

Figure 3. The effect of aligning parameter on the imaginer part of the $\mathrm{S}_{11}$ parameter

In Figure 4, the real part of $S_{12}$ is plotted from $1 \mathrm{GHz}$ to $100 \mathrm{GHz}$ with all aligned parameters listed in Table 1. It can be seen in Figure 2 that the real part of $S_{11}$ steadily increments as the frequency increase. Align parameter, which has a value of 0.4 has an effect on the imaginer part of the $S_{11}$ parameter around $20 \mathrm{GHz}$, and it has no effect on other aligned parameters.

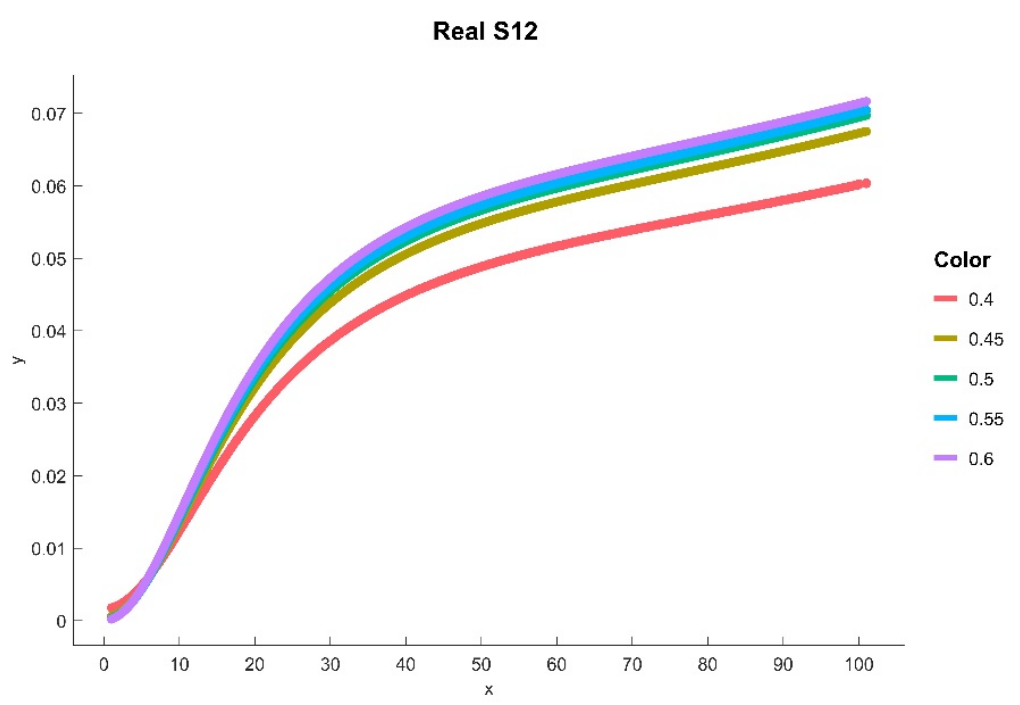

Figure 4. The effect of aligning parameter on the real part of the $S_{12}$ parameter

In Figure 5, the imaginer part of $S_{12}$ is plotted from $1 \mathrm{GHz}$ to $100 \mathrm{GHz}$ with all align parameters listed in Table 1. It can be seen in Figure 5 that the imaginer part of $S_{12}$ increments as the frequency increase. Align parameter, which has a value of 0.4 has an effect on the real part of the $\mathrm{S}_{12}$ parameter, and it does not affect other aligned values. 
Imaginer S12

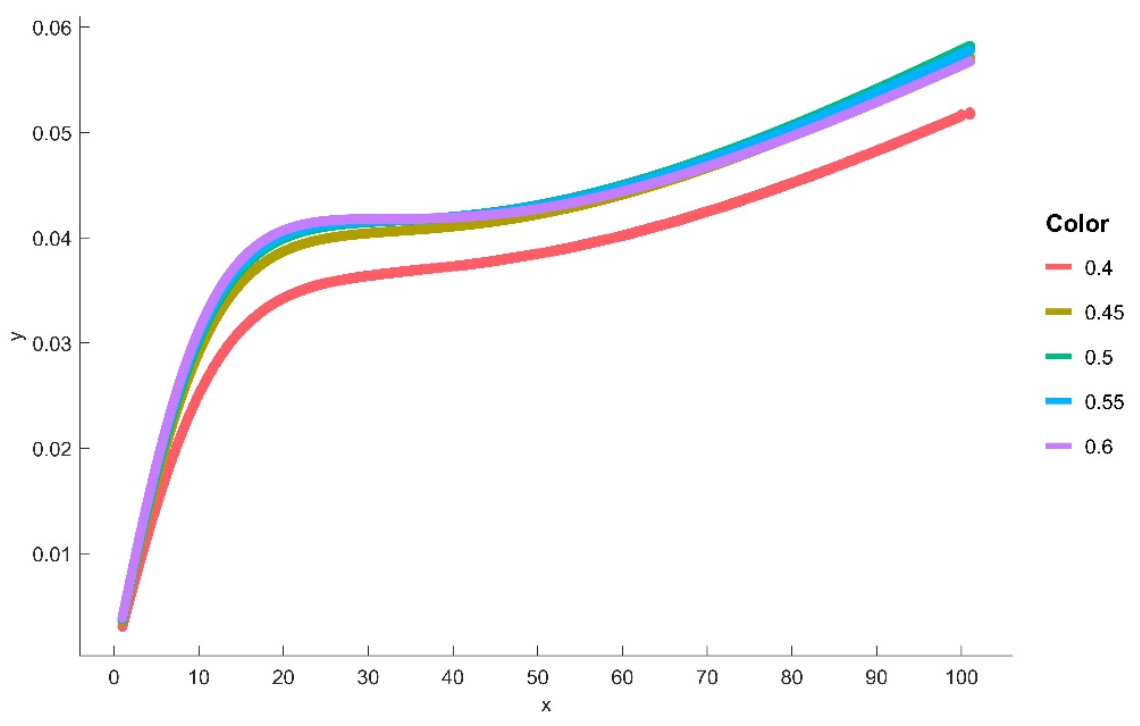

Figure 5. The effect of aligning parameter on the imaginer part of the $S_{12}$ parameter

In Figure 6, the real part of $S_{21}$ is plotted from $1 \mathrm{GHz}$ to $100 \mathrm{GHz}$ with all aligned parameters listed in Table 1. It can be seen in Figure 2 that the real part of $S_{11}$ steadily decrements until 50 $\mathrm{GHz}$, and then its sutures. All align parameter values has almost no effect on the real part of the $\mathrm{S}_{21}$ parameter.

Real S21

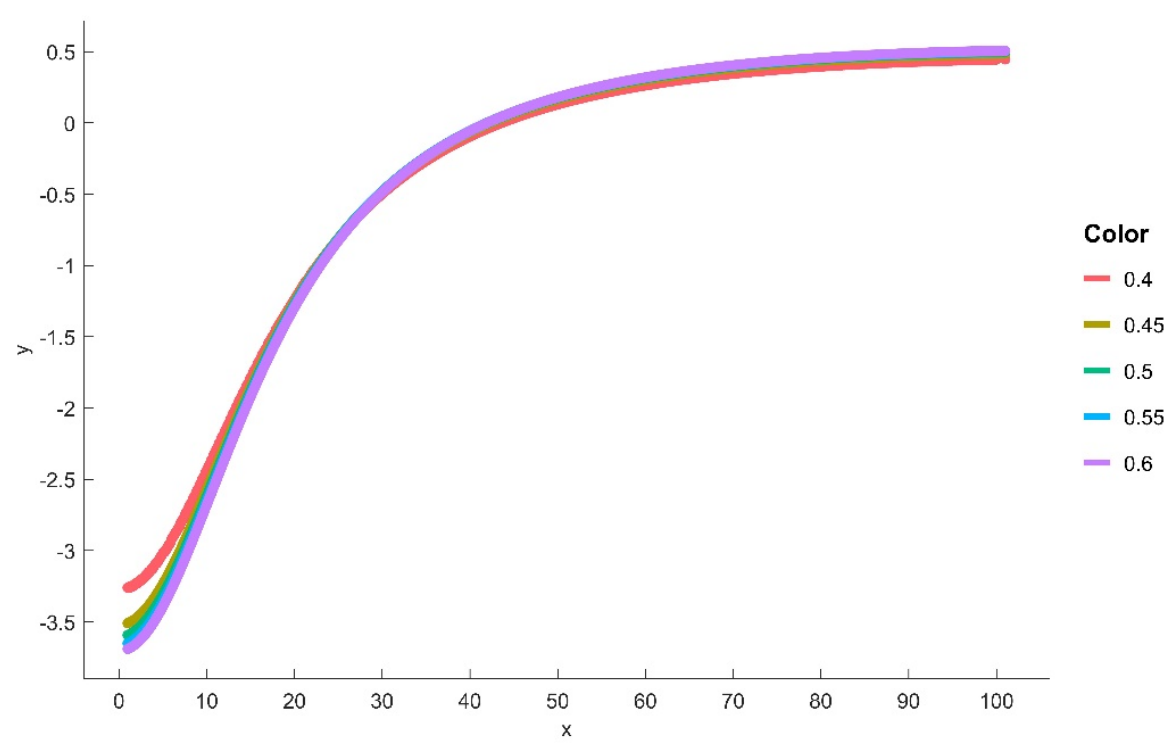

Figure 6. The effect of aligning parameter on the real part of the $S_{21}$ parameter

In Figure 7, the imaginer part of $S_{21}$ is plotted from $1 \mathrm{GHz}$ to $100 \mathrm{GHz}$ with all aligned parameters listed in Table 1. It can be seen in Figure 7 that the imaginer part of $S_{21}$ increments until frequency reaches $20 \mathrm{GHz}$ and it drops as frequency increases. Align parameter, which has a value of 0.4 has an effect on the imaginer part of the $S_{21}$ parameter around $20 \mathrm{GHz}$, and it has no effect on other aligned parameters. 
Imaginer S21

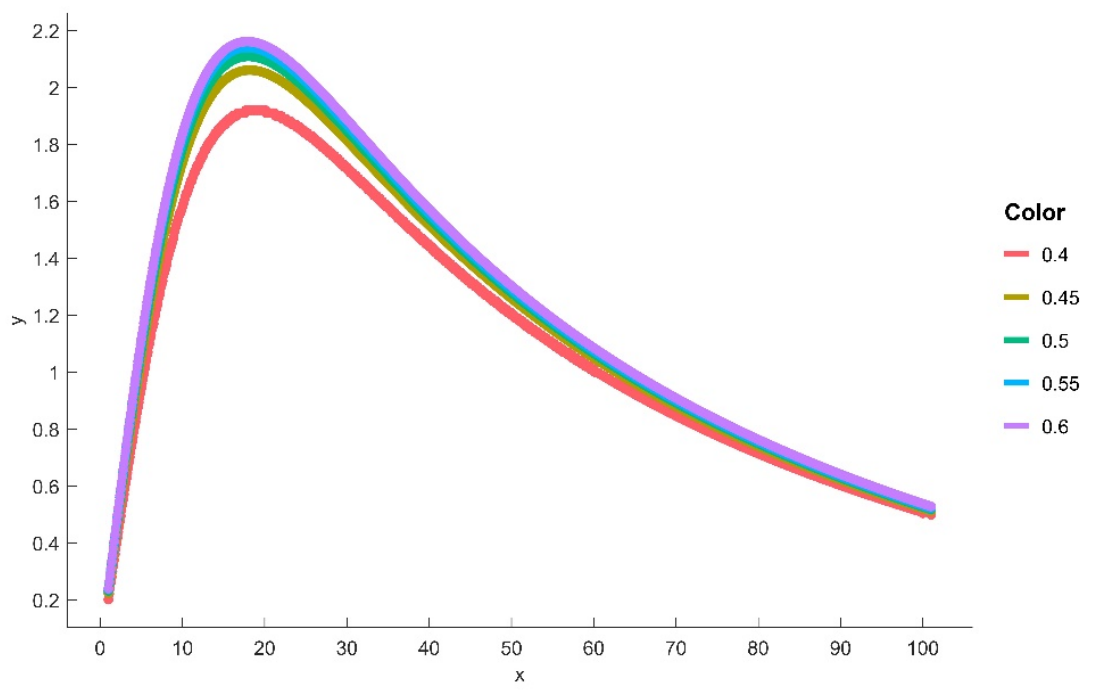

Figure 7. The effect of aligning parameter on the imaginer part of the $S_{21}$ parameter

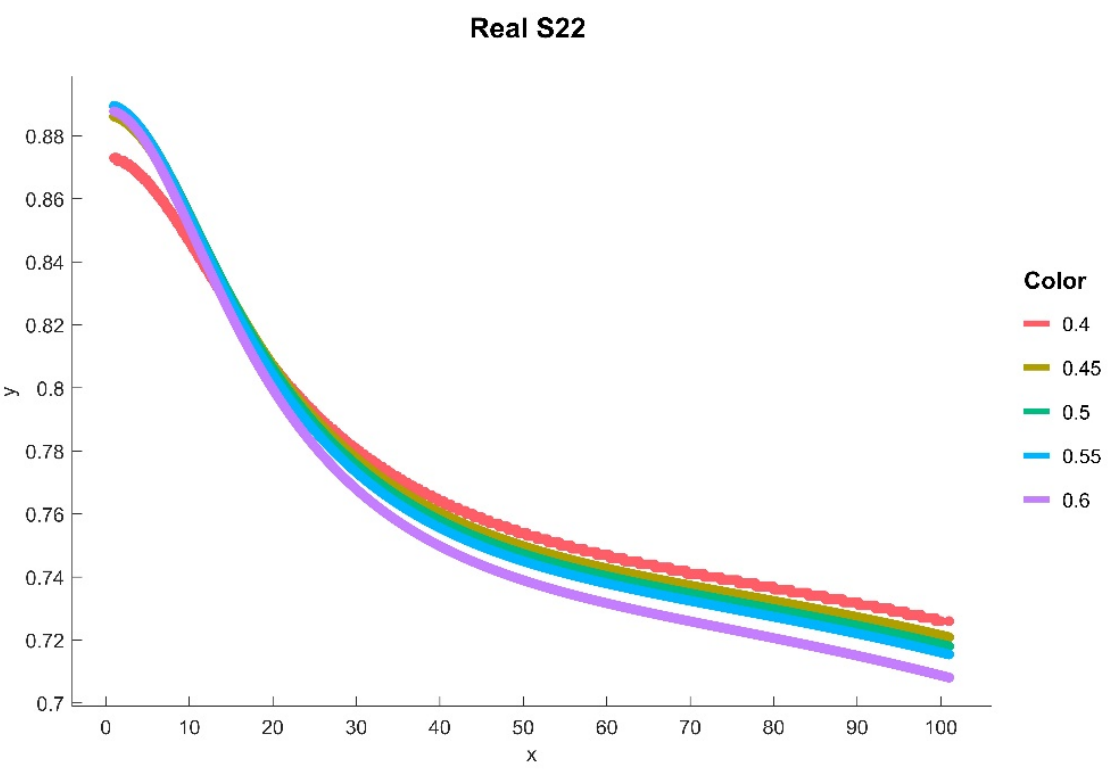

Figure 8. The effect of aligning parameter on the real part of the $S_{22}$ parameter

In Figure 8, the real part of $\mathrm{S}_{22}$ is plotted from $1 \mathrm{GHz}$ to $100 \mathrm{GHz}$ with all aligned parameters listed in Table 1. It can be seen in Figure 8 that the real part of $S_{22}$ steadily decreases as the frequency increase. All align parameter values has almost a limited effect on the real part of the S22 parameter. 


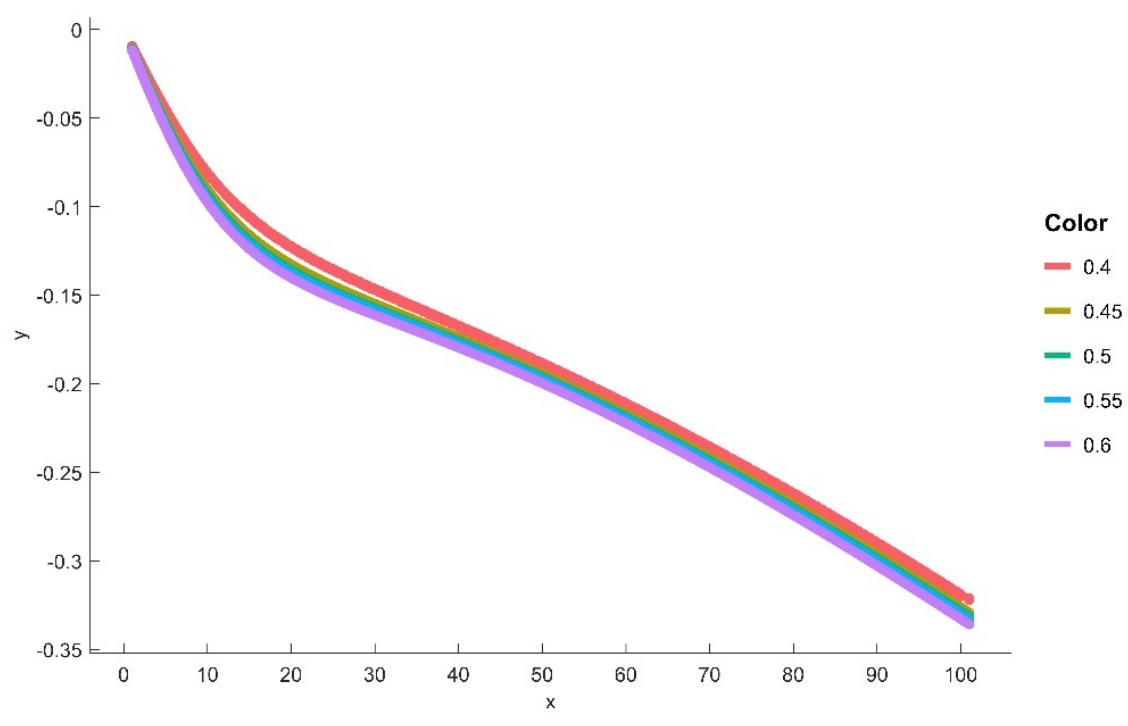

Figure 9. The effect of aligning parameter on the imaginer part of $S_{22}$ parameter

In Figure 9, the imaginer part of $S_{22}$ is plotted from $1 \mathrm{GHz}$ to $100 \mathrm{GHz}$ with all aligned parameters listed in Table 1. It can be seen in Figure 9 that the imaginer part of $S_{22}$ increments until as frequency increase. All align parameter values has almost a limited effect on the imaginer part of the $S_{22}$ parameter.

\section{CONCLUSIONS}

In this paper, two-port $\mathrm{S}$ parameters analysis of pHEMT structure is realized from $1 \mathrm{GHz}$ to $100 \mathrm{GHz}$. 8 different both real and imaginer part of S parameters are plotted from Figure 2 to Figure 9. In all figures, a brief explanation about the change of the $\mathrm{S}$ parameter concerning frequency is given. Also, the effect of the align parameter on S parameters is explained in detail. In conclusion, the effect of the align parameter, which has a value of 0.4 differs from other align parameter values such as $0.45,0.5,0.55$ and 0.6 on $\mathrm{S}$ parameters of pHEMT structure. The parameter range of the align parameter can be scaled up, and further analysis can be realized. An optimization algorithm, which maximizes both real and imaginer part of $\mathrm{S}_{21}$ while minimizing the both real and imaginer part of $S_{11}$ and $S_{22}$ parameter would help circuit designers for satisfying low noise amplifier circuit performance criteria.

\section{REFERENCES}

[1] Demirel, S. and Güneş, F. (2013). Performance characterization of a microwave transistor for the maximum output power and the required noise. IET Circuits, Devices \& Systems, 7(1): 9-20.

[2] Güneş, F. and Çetiner, B.A. (1988). Smith chart formulation of performance characterization for a microwave transistor. IEE Proceedings - Circuits, Devices and Systems, 145(6): 419-428.

[3] Güneş, F., Güneş, M. and Fidan, M. (1994). Performance characterization of a microwave transistor. IEE Proceedings-Circuits, Devices and Systems, 141(5): 337-344.

[4] Güneş, F., Türker, N. and Gürgen, F. (2007). Signal-noise support vector model of a microwave transistor. International Journal of RF and Microwave Computer-Aided Engineering, 17(4): 404-415. 
[5] Ahmad, M., Butt, H. T., Tauqeer, T. and Missous, M. (2012). DC characterization of InGaAs/InAlAs/InP based pseudomorphic HEMT (pHEMT). Paper presented at The Ninth International Conference on Advanced Semiconductor Devices and Microsystems.

[6] Ahmad, N., Arshad, S., and Missous, M. (2010). New InP based pHEMT double stage differential to singleended MMIC low noise amplifiers for SKA. Paper presented at The Eighth International Conference on Advanced Semiconductor Devices and Microsystems.

[7] Arshad, S., Mohiuddin, M., Bouloukou, A., and Missous, M. (2008). Physical Modelling of the Kink Effect in Strained InGaAs/InAlAs pHEMTs. Paper presented at the 2008 International Conference on Advanced Semiconductor Devices and Microsystems.

[8] Aziz, A. A., and Missous, M. (1996). InGaP/InGaAs/GaAs pseudomorphic HEMT grown by solid source MBE. Paper presented at the Proceedings of EDMO '96.

[9] Aziz, A. A., and Missous, M. (1997). Fabrication and characterization of AlGaAs/InGaAs/GaAs pseudomorphic HEMT with in-situ epitaxial aluminum grown by MBE. Paper presented at the IEEE MTT/ED/AP/LEO Societies Joint Chapter United Kingdom and the Republic of Ireland Section. 1997 Workshop on High Performance Electron Devices for Microwave and Optoelectronic Applications.

[10] Güneş, F., and Demirel, S. (2016). Performance characterization of a microwave transistor subject to the noise and matching requirements. International Journal of Circuit Theory and Applications, 44(5): 1012-1028.

[11] Satılmış, G., Güneş, F., and Mahouti, P. (2019). Calculation of Scattering Parameters for a MESFET Transistor. Paper presented at the 4th International Conference On Advances in Natural \& Applied Sciences.

[12] Satılmış, G., Güneş, F., and Mahouti, P. (2020). Physical parameter-based data-driven modeling of small signal parameters of a metal-semiconductor field-effect transistor. Int J Numer Model El., e2840.

[13] Gao, J. (2015). Heterojunction Bipolar Transistors for Circuit Design: Microwave Modeling and Parameter Extraction. Wiley.

[14] Abdullah, M.R.R.B. (2018). GaAs/AlAs ASPAT Diodes for Millimetre and Sub-Millimetre Wave Applications, Ph.D., The University of Manchester (United Kingdom), Ann Arbor, 11015556.

[15] Devabhaktuni, V.K., Yagoub, M.C.E., Fang, Y., Xu, J. and Zhang, Q.Z. (2001). Neural networks for microwave modeling: Model development issues and nonlinear modeling techniques. International Journal of RF and Microwave Computer-Aided Engineering, 11(1): 4-21.

[16] Jegadheesan, V. and Sivasankaran, K. (2017). RF stability performance of SOI junctionless FinFET and impact of process variation, Microelectronics Journal, 59: 15-21.

[17] Jubadi, V.M., Zawawi, M.A.M. and Missous, M. (2014). Simulative study on physical modeling of submicrometer highly-strained In0.8Ga0.2As/AlAs resonant tunneling diode, The Tenth International Conference on Advanced Semiconductor Devices and Microsystems, 1-4.

[18] Dragica Vasileska, S. M. G. (2006). Computational Electronics: Morgan \&Claypool Publishers.

[19] PHEMT High Frequency Analysis. 2020 [cited 2020 17.01.2020]; Available from: https://silvaco.com/examples/tcad/section10/example6/index.html 